Gdańsk 2020, Nr. 43

https://doi.org/10.26881/sgg.2020.43.06

Izabela Kujawa

Universität Gdańsk / Uniwersytet Gdański

ORCID: 0000-0002-8672-2324

\title{
Linguistische Diskursforschung in Polen. Stand und Perspektiven
}

\begin{abstract}
Der vorliegende Beitrag konzentriert sich auf diskurslinguistische Forschungstraditionen der Polonistik und Germanistik in Polen sowie auf exemplarisch ausgewählte Studien zur Diskurslinguistik. Der Beitrag setzt sich zum Ziel, sich mit den theoretischen und methodologischen Aspekten der linguistischen Diskursanalyse in Polen auseinanderzusetzen sowie wichtige Ansätze der Diskursforschung und Perspektiven ihrer künftigen Entwicklung zu präsentieren.

Schlüsselwörter: diskurslinguistische Forschungstraditionen, Diskurslinguistik, Diskursanalyse, Diskursforschung

Linguistic research on discourse in Poland. The state of research and its perspectives. - This paper focuses on discursive research traditions of Polish and German studies in Poland, as well as on selected studies in discourse linguistics. The article aims at discussing some theoretical and methodological aspects of linguistic discourse analysis in Poland, as well as at presenting important approaches to discourse research and perspectives of its further development.
\end{abstract}

Keywords: discursive linguistic research traditions, discourse linguistics, discourse analysis, discourse research

\section{Zum Begriff des Diskurses. Vielfalt an Diskursdefinitionen und methodischen Ansätzen}

In der zweiten Hälfte des 20. Jahrhunderts und besonders gegen dessen Ende hat der Begriff Diskurs ,einen Diffusionsprozess erfahren, indem er aus je unterschiedlichen wissenschaftlichen Bestimmungen in die alltägliche Wissenschaftssprache übergegangen ist" (Ehlich 2007: 116). Gerade dadurch ist der Diskurs zu einem Schlüsselkonzept geworden, dessen Wert und Bedeutungsgrenzen durch den Gebrauch jeweils bestimmt werden (Kujawa 2014: 25). Mit dem Begriff Diskurs ist eine gewisse Mannigfaltigkeit verbunden. Heinemann (2009: 366) bezeichnet den Diskurs als „Diskurs-Chamäleon“. Nicht ohne Grund gehört der Diskurs zu denjenigen Begriffen, die ,ihre kulturelle Ubiquität semantischer Offenherzigkeit verdanken“" (Plumpe 1988: 330), d. h. über ein breites Bedeutungsspektrum verfügen.

Auch die Sprachwissenschaft rezipiert den Begriff jeweils nach Forschungsprogramm und -perspektive. „Irreführend wäre die Behauptung, dass man auch innerhalb der 
Sprachwissenschaft einen konsensfähigen Diskursbegriff erarbeitet habe" (Czachur 2011:41). Es wird dabei vorgeschlagen, den Diskurs nicht als eine mehrdeutige, sondern eine unscharfe Kategorie zu definieren, deren Grenzen nicht für immer gesetzt sind, sondern jeweils bestimmt werden müssen (vgl. Witosz 2016: 20). Dies erlaubt es, den Diskurs als eine relative Kategorie zu betrachten. Seine Bedeutung, sein Umfang und Gebrauch sind durch ein konkretes theoretisches Paradigma und konkrete Forschungstraditionen determiniert (vgl. ebd.: 21).

Die Diskurslinguistik und Methodologie der Diskursanalyse in Polen sind differenziert und unterscheiden sich je nach der Forschungsperspektive der Polonisten oder anderer Philologen. Die polnischen Neuphilologen, die Repräsentanten u. a. der deutschen, anglo-amerikanischen oder französischen Philologien, prägen in ihrer Diskursforschung Perspektiven und Methodologien anderer diskurslinguistischer Forschungstraditionen, deren Sprache sie untersuchen. So werden die linguistischen Auffassungen des Diskurses (als einer interdisziplinär etablierten Kategorie) mit den Konzeptionen der Diskurstheorien aus anderen Ländern konfrontiert. Die jeweils differenzierten Herangehensweisen an den Diskursbegriff weisen wesentliche Unterschiede auf und erlauben es, den Diskurs aus mehreren Perspektiven zu erforschen.

Der vorliegende Beitrag konzentriert sich auf diskurslinguistische Forschungsbereiche der Polonistik und Germanistik in Polen, auf exemplarisch ausgewählte Studien zu Diskurslinguistik. Der Beitrag setzt sich zum Ziel, sich mit den theoretischen und methodologischen Aspekten der linguistischen Diskursanalyse in Polen auseinanderzusetzen sowie wichtige Ansätze der Diskursforschung und Perspektiven ihrer künftigen Entwicklung zu präsentieren. Kontrastive Analysen der polnischen und deutschen Diskursforschung wären eine große Herausforderung für die Linguisten, die sich mit diesem Forschungsbereich beschäftigen. Entscheidend dabei wäre es, ,in die Begrifflichkeit der fremdsprachigen Wissenschaftskreise durchzudringen “ und gleichzeitig „Forschungstraditionen der zu untersuchenden Bereiche zu recherchieren und über diese zu reflektieren "(Hanus 2015: 192) und sie als Kategorien der Untersuchung zu betrachten.

\section{Diskurs in der polonistischen Diskursforschung in Polen. Konzeptualisierungen}

Die Diskurslinguistik in Polen ist ein sich dynamisch entwickelnder Bereich der Linguistik mit einem großen Erkenntnispotenzial. Sie verdankt ihre disziplinäre Etablierung innerhalb der germanistischen und polonistischen Linguistik der Tatsache, dass bisher noch kein Forschungsprogramm herausgearbeitet wurde, das methodologisch den interdisziplinären Herausforderungen gerecht werden könnte (vgl. Czachur 2011: 185).

Der Terminus Diskurs gelangte in die polnische Textlinguistik mit den Arbeiten des französischen Semiologen Émile Benveniste ${ }^{1}(1902-1976)$ und mit seinem Diskursverständnis: discours verstanden als ,ein Text, dessen Struktur auf seine Immersion in der ihn begleitenden Situation verweist" (Witosz 2010: 169).

Einen besonderen Einfluss auf die Konzeptualisierung des Diskurses in der polonistischen Forschung hatten zahlreiche Monographien und Studien, die sich mit unterschiedlichen

1 Vgl. Benveniste, Émile (1966): Problèmes de linguistique générale. Paris. 
Aspekten der Kommunikation und des Textes befassten, wobei der Text als ein Kommunikationsereignis in einem kulturell-soziologischen Kontext betrachtet wurde. Zu erwähnen sind dabei vor allem Arbeiten zu folgenden Themen:

- Relationen zwischen Text und Diskurs (Bartmiński / Niebrzegowska-Bartmińska 2009, Żydek-Bednarczuk 2005),

- Analyse des Diskurses aus der Sicht der französischen Linguistik (Grzmil-Tylutki 2007, 2010),

- kritische Diskursanalyse (Critical Discourse Studies CDA) (Duszak / Fairclough 2008, Duszak 1998).

Zwei wesentliche Forschungstraditionen haben die Konzeptualisierung der Diskurskategorie und die Diskursanalyse in der polonistischen Linguistik geprägt (Witosz 2012: 61):

- die Textlinguistik (Textdefinition mit einem pragmatischen Charakter),

- die Funktionalstilistik (Stilforschung, Stildefinition mit einem kulturanthropologischen Charakter).

Die polnische Textlinguistik und Textsortenlinguistik sind mit der polonistischen Stilforschung eng verbunden, die sich als eine Disziplin mit allen modernen Teilbereichen der Linguistik in Beziehung setzt (vgl. Hanus 2015: 192). Dem Stil wird eine dominierende Stellung zugeschrieben, was eine entsprechende Erklärung in der polnischen Textlinguistik hat, wo die Relationen zwischen Text und Stil im Kontext der Funktionalstile untersucht werden (vgl. Hanus 2015: 196). So werden in der polonistischen Linguistik Stil, Text, Gattung und Diskurs in gegenseitigen Beziehungen untersucht und als eine „Kollektion“ betrachtet, als ein „Erkenntnisraum, in dem die in Betracht gezogenen Begriffe aufeinander bezogen sind“ (Wojtak 2011: 69). Im Rahmen der polonistischen Forschung wurde der Terminus Diskursstilistik von Gajda (2005) vorgeschlagen. Zu erwähnen sind in diesem Zusammenhang die Arbeiten polnischer Linguisten wie: Dobrzyńska (1996), Gajda / Balowski (1996), Witosz (2009), Wojtak (2011), Bartmiński / Niebrzegowska-Bartmińska (2009).

Ähnlich wie in der germanistischen Forschungstradition wird der Begriff Diskurs auch in der polonistischen Tradition unterschiedlich definiert und verstanden. Die Vielfalt an Herangehensweisen bewirkt, dass der innerhalb der Polonistik erarbeitete Begriff des Diskurses sehr allgemein ist. Als eine verallgemeinernde Kategorie betrachtet den Diskurs Witosz in ihren Arbeiten von 2010 und 2012. Diskurs ist eine „Menge von Äußerungen [...], die aus bestimmten sozialen, z. B. Lehrerdiskurs, Verwaltungsdiskurs, oder ideologischen, z. B. feministischer Diskurs, liberaler Diskurs, Positionen konstruiert werden“" (Witosz 2010: 175).

Grzegorczykowa (2008: 41) beschreibt drei Dimensionen des Diskurses, nämlich:

- Diskurs als eine logisch aufgebaute Aussage,

- Diskurs als ein Kommunikationsereignis,

- Diskurs als eine Meinungsäußerung.

Einige Forscher belegen, dass die Konzeption des Diskurses viele Gemeinsamkeiten mit der des Textes hat (vgl. Dobrzyńska 2009, Witosz 2009). Witosz (2010: 178) betont, dass die Kategorie des Diskurses und die um sie aufgebauten Voraussetzungen nur die Forschungsperspektive ändern:

„Methodologische Reorientierung (diskursive Orientierung) führt in die Konzeption des Textes keine völlig neuen, bisher nicht reflektierten Parameter ein, sie bestimmt jedoch neue Konfigurationen 
im Bereich der schon bekannten Konfigurationen und gestaltet gleichzeitig die Felder der Textdeterminanten um und schließt die Kategorie des Textes in den breiten Raum sozialer Interaktionen ein“ (Witosz 2010: 178).

Betrachtet man die Untersuchung des Diskurses als eine Erweiterung der Textuntersuchung (mit Berücksichtigung aller stilistischen Fragen), so soll der Diskurs auch in Bezug auf die Diskursstilistik analog erforscht werden (vgl. Witosz ebd.). Eine andere Perspektive der Diskursforschung dagegen lässt beide Kategorien: Diskurs und Text als Synonyme betrachten und untersuchen (vgl. Boniecka 1999, Żydek-Bednarczuk 2005). Darüber hinaus plädiert Wojtak (2011: 71) dafür, Begriffe wie Diskurs, Stil, Gattung und Text aufeinander zu beziehen, wobei der Diskurs als eine übergeordnete Kategorie fungiert und ein Ausgangspunkt für Überlegungen zu den Relationen zwischen den vier genannten Begriffen ist. Der Diskurs wird durch solche Parameter definiert, die in der Gattung / Textsorte enthalten sind. Der Stil dagegen wird als Bestandteil der Gattungsmuster / Textsortenmuster betrachtet und soll als ein Konkurrenzbegriff zum Diskurs verstanden werden (vgl. Wojtak 2011: 71, Hanus 2015: 199).

Der Begriff des Diskurses wird aber gegenwärtig in der Polonistik vor allem als Ergebnis der Kommunikationspraxis einer bestimmten Gruppe oder Gesellschaft verstanden, als „eine Norm und Strategie, die im Prozess der Textproduktion und Aussage angewandt wird. Die Grundlage dieser Strategie bilden die sozialen und kulturellen Muster, die diese Norm ausmachen, und deren Ergebnis ein Text oder eine Aussage von bestimmten Gattungsmerkmalen ist" (Labocha 1996: 51). In dem Sinne ähnelt die polonistische Betrachtungsweise des Diskurses der germanistischen, wobei die germanistische Diskursforschung an Foucaults Diskurstheorien stark orientiert ist.

\section{Diskurs in der germanistischen Diskursforschung in Polen}

In der germanistischen Forschungstradition werden solche Begriffe wie Diskurs und Stil nicht aufeinander bezogen. Die germanistische Diskursforschung knüpft häufig an die Werke von Michel Foucault (1926-1984) an, besonders an seine Studien zu den Beziehungen zwischen Wissen und Macht. In Anlehnung an die Diskurstheorie des französischen Philosophen entstanden zwei Richtungen der germanistischen Diskursforschung: bistorisch-semantische Diskursforschung bzw. Diskursgeschichte und die Kritische Diskursanalyse. Die erste beschäftigt sich mit der Analyse von Schlüssel- und Schlagwörtern, Begriffen, Metaphern, Argumentationsmustern etc. und erfasst Wissensformationen und Denkschemata in einem bestimmten Zeitraum. Die zweite, kritisch orientierte Diskursforschung wird auf die Erforschung von Machtverhältnissen sowie (expliziten und impliziten) Machtpraktiken fokussiert.

Die folgenden Diskursdefinitionen mit einer unterschiedlichen Ausprägung (semantisch oder kritisch orientiert) präsentieren jeweils andere Aspekte des Diskurses:

„Zu einem Diskurs gehören alle Texte, die sich mit einem als Forschungsgegenstand gewählten Gegenstand, Thema, Wissenskomplex oder Konzept befassen, untereinander semantische Beziehungen 
aufweisen und/oder in einem gemeinsamen Aussage-, Kommunikations-, Funktions- oder Zweckzusammenhang stehen, den als Forschungsprogramm vorgegebenen Eingrenzungen in Hinblick auf Zeitraum/Zeitschnitte, Areal, Gesellschaftsausschnitt, Kommunikationsbereich, Texttypik und andere Parameter genügen, und durch explizite und implizite [...] Verweisungen aufeinander Bezug nehmen bzw. einen intertextuellen Zusammenhang bilden“" (Busse / Teubert 1994: 14).

Die kritisch orientierte Diskursauffassung setzt sich zum Ziel, das (jeweils gültige) Wissen der Diskurse zu ermitteln, den konkreten jeweiligen Zusammenhang von Wissen und Macht zu erkunden und einer Kritik zu unterziehen:

„Texte sind Bestandteile bzw. Fragmente von Diskurssträngen (=Abfolgen von Diskursfragmenten mit gleicher Thematik), die sich auf verschiedenen Diskursebenen (=Orte, von denen aus gesprochen wird, also Wissenschaft, Politik, Medien, Alltag etc.) bewegen und in ihrer Gesamtheit den Gesamtdiskurs einer Gesellschaft ausmachen, den man sich als ein großes, wucherndes Gewimmel vorstellen kann; zugleich bilden die Diskurse (bzw. dieses gesamte diskursive Gewimmel) die jeweiligen Voraussetzungen für den weiteren Verlauf des gesamtgesellschaftlichen Diskurses“( Jäger 1999: 117).

„Diskursanalyse bezieht sich sowohl auf Alltagswissen, das über Medien, alltägliche Kommunikation, Schule, Familie etc. vermittelt wird, wie auch auf dasjenige (jeweils gültige) Wissen, das durch die Wissenschaften produziert wird“ (Jäger 1994: 1).

„Diskurse sind eine prinzipiell offene Menge von thematisch zusammenhängenden und aufeinander bezogenen Äußerungen. Es handelt sich nicht um objektiv gegebene und (streng) gegeneinander abgegrenzte Komplexe, sondern um Zusammenhänge, die eine Kommunikationsgemeinschaft im gesellschaftlich-historischen Prozess als geistige Ordnungsgröße konstituiert, vor deren Hintergrund einzelne Äußerungen und Texte produziert und rezipiert werden oder, um eine modische Formulierung zu benutzen, in die sie sich einschreiben. [...] Keine Äußerung und kein Text (ent)steht unabhängig von anderen, als rein individuelle Kreation, die lediglich und unmittelbar auf das Sprachsystem bezogen wäre, dessen Regeln gewissermaßen nur applizierte, sondern ist immer eingebunden in das Universum von bereits Gesagtem“" (Adamzik 2004: 254).

Innerhalb der germanistischen Diskursforschung werden Foucaults Thesen u. a. von Warnke / Spitzmüller (2008, 2011), Spitzmüller (2005), Wengeler (2003), Busse / Teubert (1994), Jung (1996), Jäger $(1994,1999)$, Wodak (2005) vertreten und in die germanistische Diskursforschung in Polen übertragen.

Die sich im deutschsprachigen Raum deutlich abzeichnende Rezeption des poststrukturalistischen Diskurskonzepts beeinflusst auch die Arbeiten der polnischen Germanisten. Gegenstand ihres Interesses ist die Analyse diskursiver Weltbilder (verstanden als entstandene Wissensformationen zu einer bestimmten Zeit in einem bestimmten sozialen und kulturellen Zusammenhang, in einer bestimmten Gesellschaft) und Wissenstransfer unter den Diskursteilnehmern (vgl. Czachur 2011: 53).

Die in letzter Zeit entstandene Online-Kommunikation und die sozialen Medien eröffneten neue Wege der Diskursforschung, nämlich die Erforschung des Wissenstransfers und der Wissenskonstituierung in den Online-Diskursen, die aufgefasst werden als „thematisch gebundene, multimodal konstituierte und transmedial vermittelte Aussageketten [...], die gegenwärtige (Wissens-)Gesellschaft [motivieren], im Rezeptionsprozess Wissen über ein bestimmtes kulturelles Artefakt zu bilden" (Opiłowski 2018: 284). 
Die Spielräume in der digitalen Umgebung und die diskursiven Blog-Interaktionen, d. h. die Blogosphäre, deren Mikro-Ebene einer Blog-Interaktion in einen Diskurs eingebunden wird, erforscht Joanna Pędzisz in ihrer Monographie (2017) über das Profil des OnlineDiskurses in Blog-Interaktionen.

Neue Herausforderungen und Forschungsfelder für die linguistische Diskursanalyse bieten kontrastive Studien im deutsch-polnischen Bereich an. Erwähnenswert sind dabei die Monographien von Waldemar Czachur (2011) über die kontrastive Diskursanalyse deutscher und polnischer Medien, von Dorota Miller (2014) über Emotionalität und Wertung im Diskurs, oder die Fallstudien zum deutsch-polnischen medialen Gegendiskurs von Dorota Kaczmarek (2018), sowie auch der von Ryszard Lipczuk, Dorota Misiek, Jürgen Schiewe und Werner Westphal herausgegebene Konferenzband (2010), der teilweise der kontrastiven Diskursforschung gewidmet ist.

Neben den kontrastiven Analysen gibt es auch einige Monographien, deren Verfasser sich mit medialen Diskursen zu ausgewählten Themen beschäftigen, z. B. mit dem politischen Diskurs zu Integration und Multikulturalismus in Deutschland (Kujawa 2014) oder mit dem rechtsextremistischen Straßendiskurs in Deutschland (Kumięga 2013).

Mit den didaktischen Perspektiven der Diskursforschung und Methoden der Diskursanalyse aus didaktischer Sicht befassen sich Czachur / Kulczyńska / Kumięga (2016), die der Frage nachgehen, wie der Diskurs für die akademische Didaktik analysiert und definiert werden sollte.

Im Bereich der Diskursforschung in Polen gibt es zahlreiche Projekte, die mit Erfolg realisiert werden, sowie etablierte Zentren für die Diskursforschung. 2011 entstand an der Universität Rzeszów das wissenschaftlich-didaktische Zentrum: Ośrodek Badawczo-Dydaktyczny i Transferu Wiedzy „Tekst-Dyskurs-Komunikacja“, gegründet von Zofia Bilut-Homplewicz, Anna Hanus, Agnieszka Mac, Dorota Miller, Marta Smykała und Iwona Szwed. Die wissenschaftliche Tätigkeit der Forscherinnen konzentriert sich auf die Text- und Diskursforschung sowie auf die Wissenspopularisierung, Didaktik und breit verstandene Zusammenarbeit mit anderen, auch ausländischen, Forschern. Im Rahmen der Zusammenarbeit mit dem Germanistischen Institut der Universität Warschau wird die Fachzeitschrift text und diskurs - tekst i dyskurs herausgegeben, die sich als ,eine interdisziplinäre und fachübergreifende Plattform für den Gedankenaustausch, für die modellhaften Lösungen im Bereich der Textund Diskurslinguistik “2 versteht und für alle philologischen Fachrichtungen zugänglich ist. Die erste Ausgabe der Zeitschrift text und diskurs - tekst i dyskurs erschien 2008, der Herausgeber und Leiter der Redaktion ist bis jetzt Waldemar Czachur. Seit 2011 wird die Zeitschrift am Germanistischen Institut der Universität Warschau und am Lehrstuhl für Angewandte Linguistik der Universität Rzeszów redigiert.

Zu nennen ist auch die seit 2011 bei Peter Lang Verlag erscheinende Schriftenreihe Studien zur Text-und Diskursforschung, herausgegeben von Zofia Bilut-Homplewicz von der Universität Rzeszów und Zofia Berdychowska von der Jagiellonen-Universität in Krakau. Der erste Sammelband Text und Stil, hg. von Zofia Bilut-Homplewicz, Agnieszka Mac, Marta Smykała und Iwona Szwed, erschien im Jahre 2011.

2 http://tekst-dyskurs.eu/index.php/de/. 
Neben zahlreichen wissenschaftlichen Veröffentlichungen gibt es in Polen auch eine Vielzahl von internationalen Konferenzen zur Diskursforschung:

- „Diskurse als Mittel und Gegenstände der Germanistik. Jahrestagung und internationale wissenschaftliche Konferenz des Verbandes Polnischer Germanisten “ (an der Universität Olsztyn, 8.-10. Mai 2009);

- „Der Diskurs im Spannungsfeld von System- und angewandter Linguistik“ (eine polnischdeutsche Tagung, veranstaltet vom Institut für Germanistik der Universität Szczecin und Institut für Deutsche Philologie der Universität Greifswald; Pobierowo an der Ostsee, 15.-18. September 2009);

- „Text- und Diskurswelten in der massenmedialen Kommunikation - Zugänge zur Produktion, Rezeption und Wirkung von multimodalen Textformaten" (eine internationale Konferenz an der Universität Bydgoszcz, 18.-19. Oktober 2018).

Die germanistische Diskurslinguistik ist durch solide theoretische Grundlagen und methodologischen Pluralismus gekennzeichnet (vgl. Warnke / Spitzmüller 2009: 124). Gegenstand ihrer Analyse sind feste Diskurskomponenten, wie Text, Handlung, Wissen und Macht, deren Erforschung mehrdimensional ist - übrigens wie die Kategorie des Diskurses selbst, die unter Berücksichtigung textbezogener, handlungsbezogener, wissensbezogener und machtbezogener Dimensionen verstanden werden soll (vgl. Czachur 2011: 54-55).

Die Antwort auf die Vielfältigkeit der Methodologien war das Modell der Diskurslinguistischen Mehr-Ebenen-Analyse (DIMEAN) von Warnke / Spitzmüller (2008). Das Modell berücksichtigt alle möglichen Kategorien der linguistischen Diskursanalyse auf der Grundlage der Diskurstheorie von Michel Foucault. DIMEAN integriert grundlegende Kategorien der Diskursanalyse auf allen Ebenen: der intratextuellen, der transtextuellen und auf der Ebene der Diskursakteure, die alle erwähnten Ebenen verbindet.

\section{Fazit}

Der Überblick über die exemplarisch ausgewählten polonistischen und germanistischen Studien aus dem Bereich der Diskursforschung erlaubt die Feststellung, dass die Etablierung der Diskursanalyse in Polen ein großes Potenzial mit sich bringt. Sie soll aber interdisziplinär verstanden werden, denn sie „erfüllt die Brückenfunktion zwischen den in der fremdsprachlichen Germanistik etablierten Linguistik, Kulturwissenschaft und Literaturwissenschaft" (Czachur 2011: 188). Die germanistische Diskursforschung in Polen hat die soliden Grundlagen der Diskurslinguistik aus dem deutschsprachigen Raum. Was die polonistische Forschung an die germanistische näher bringen kann, sind die Postulate von Gajda (2005: 143), die stilistische Einheit von Texten als ein Textualitätskriterium im Sinne der Textualitätsmerkmale von de Beaugrande / Dressler (1981) zu berücksichtigen und Texte als stilistische Einheiten zu betrachten, wo der Stil die Texthaftigkeit und der Text die stilistische Einheit seiner Oberfläche voraussetzt (vgl. Fix 2007: 451). Dies ermöglicht es, die Erforschung des Diskurses als eine Erweiterung der Textuntersuchungen zu betrachten und eine entsprechende Methodologie der Analyse zu erarbeiten. 


\section{Literatur}

Adamzik, Kirsten (2004): Textlinguistik. Eine einführende Darstellung. Tübingen: Niemeyer.

Bartmiński, Jerzy / Niebrzegowska-Bartmińska, Stanisława (Hg.) (2009): Tekstologia [Textwissenschaft]. Warszawa: PWN.

Benveniste, Émile (1966): Problèmes de linguistique générales. Paris: Gallimart.

Bilut-Homplewicz, Zofia / Mac, Agnieszka / Smykała, Marta / Szwed, Iwona (Hg.) (2011): Text und Stil. Frankfurt/M.: Lang.

Boniecka, Barbara (1999): Lingwistyka tekstu: teoria i praktyka [Textlinguistik: Theorie und Praxis]. Lublin: Wydawnictwo UMCS.

Busse, Dietrich / Teubert, Wolfgang (1994): Ist Diskurs ein sprachwissenschaftliches Objekt? Zur Methodenfrage der Historischen Semantik. In: Busse, Dietrich / Hermanns, Fritz / Teubert, Wolfgang (Hg.): Begriffsgeschichte und Diskursgeschichte. Methodenfragen und Forschungsergebnisse der historischen Semantik. Opladen: Westdeutscher Verlag, 10-28.

Czachur, Waldemar (2011): Diskursive Weltbilder im Kontrast. Linguistische Konzeption und Methode der kontrastiven Diskursanalyse deutscher und polnischer Medien. Wrocław: ATUT.

Czachur, Waldemar / Kulczyńska, Agnieszka / Kumięga, Eukasz (Hg.) (2016): Jak analizowaí dyskurs? Perspektywy dydaktyczne [Wie soll der Diskurs analysiert werden? Didaktische Perspektiven]. Kraków: Universitas.

de Beaugrande, Robert / Dressler, Wolfgang Ulrich (1981): Einführung in die Textlinguistik. Tübingen: Niemeyer.

Dobrzyńska, Teresa (1996): Tekst - w perspektywie stylistycznej [Text - in stilistischer Perspektive]. In: Dies. (Hg.): Tekst i jego odmiany. Zbiór studiów [Der Text und seine Varianten. Ausgewählte Abhandlungen]. Warszawa: Instytut Badań Literackich, 125-143.

Dobrzyńska, Teresa (2009): Pojęcie tekstu. Tekst - całościowy komunikat. Tekst a/i dyskurs w perspektywie polskiej tradycji badań nad tekstem [Zum Textbegriff. Text - eine ganzheitliche Botschaft. Text und Diskurs in der Perspektive der polnischen Tradition der Texterforschung]. In: BilutHomplewicz, Zofia / Czachur, Waldemar / Smykała, Marta (Hg.): Lingwistyka tekstu w Polsce iw Niemczech. Pojęcia, problemy, perspektywy [Textlinguistik in Polen und in Deutschland. Begriffe, Probleme, Perspektiven]. Wrocław: ATUT, 35-45.

Duszak, Anna / Fairclough, Norman (2008): Krytyczna analiza dyskursu. Interdyscyplinarne podejście do komunikacji spotecznej [Kritische Diskursanalyse. Ein interdisziplinärer Zugang zur sozialen Kommunikation]. Kraków: Universitas.

Duszak, Anna (1998): Tekst, dyskurs, komunikacja międzykulturowa [Text, Diskurs, interkulturelle Kommunikation]. Warszawa: PWN.

Ehlich, Konrad (2007): Sprache und sprachliches Handeln. Pragmatik und Sprachtheorie, Prozeduren des sprachlichen Handelns, Diskurs, Narration, Text, Schrift. 3 Bände. Berlin / New York: De Gruyter.

Fix, Ulla (2007): Stil - ein sprachliches und soziales Phänomen. Beiträge zur Stilistik. Berlin: Frank \& Timme.

Gajda, Stanisław / Balowski, Mieczysław (Hg.) (1996): Styl a tekst [Stil und Text]. Opole: Wydawnictwo Uniwersytetu Opolskiego.

Gajda, Stanisław (2005): Tekst/dyskurs oraz jego analiza i interpretacja [Text/Diskurs, seine Analyse und Interpretation]. In: Krauz, Maria / Gajda, Stanisław (Hg.): Wspótczesne analizy dyskursu [Gegenwärtige Diskursanalysen]. Rzeszów: Wydawnictwo Uniwersytetu Rzeszowskiego, 11-20. 
Grzegorczykowa, Renata (1998): Głos w dyskusji o pojęciu tekstu i dyskursu [Zum Text- und Diskursbegriff: ein Diskussionsbeitrag]. In: Bartmiński, Jerzy / Boniecka, Barbara (Hg.): Tekst. Problemy teoretyczne [Text. Theoretische Fragen]. Lublin: Wydawnictwo UMCS, 37-44.

Grzegorczykowa, Renata (2008): Wstęp do jezzykoznawstwa [Einführung in die Sprachwissenschaft]. Warszawa: PWN.

Grzmil-Tylutki, Halina (2007): Gatunek w świetle francuskiej teorii dyskursu [Die Gattung aus der Perspektive der französischen Diskurstheorie]. Kraków: Universitas.

Grzmil-Tylutki, Halina (2010): Francuska lingwistyczna teoria dyskursu. Historia, tendencje, perspektywy [Die französische linguistische Diskurstheorie. Geschichte, Tendenzen, Perspektiven]. Kraków: Universitas.

Hanus, Anna (2015): Wie werden Stil, Gattung, Text und Diskurs in der Polonistik positioniert? Grundbegriffe der Text- und Diskursforschung im deutsch-polnischen Vergleich - dargestellt aus dem Blickwinkel eines germanistischen Forschers. In: Studia Germanica Gedanensia 33, 179-191.

Heinemann, Wolfgang (2009): Anmerkungen zum Paradigmenwechsel in der Sprachwissenschaft. In: Czachur, Waldemar / Czyżewska, Marta / Frączek, Agnieszka (Hg.): Wort und Text. Bestandsaufnahme und Perspektiven. Warszawa: Universitas Varsoviensis, 28-39.

Jäger, Siegfried (1994): Text-und Diskursanalyse. Eine Anleitung zur Analyse politischer Texte. Duisburg: DISS

Jäger, Siegfried (1999): Kritische Diskursanalyse. Eine Einführung. Duisburg: DISS.

Jung, Martin (1996): Linguistische Diskursgeschichte. In: Böke, Karin / Jung, Martin / Wengeler, Martin (Hg.): Öffentlicher Sprachgebrauch. Opladen: De Gruyter, 453-472.

Kaczmarek, Dorota (2018): Binationale Diskursanalyse. Grundlagen und Fallstudien zum deutschpolnischen medialen Gegendiskurs. Łódź: Wydawnictwo Uniwersytetu Łódzkiego.

Kujawa, Izabela (2014): Der politische Diskurs als Gegenstand der linguistischen Analyse am Beispiel der Integrationsdebatte in Deutschland 2006-2010. Frankfurt/M.: Lang.

Kumięga, Łukasz (2013): Rechtsextremistischer Straßendiskurs in Deutschland. Studien zur Text- und Diskursforschung. Frankfurt/M.: Lang

Labocha, Janina (1996): Tekst, wypowiedź, dyskurs [Text, Äußerung, Diskurs]. In: Gajda, Stanisław / Balowski, Mieczysław (Hg.): Styl a tekst [Stil und Text]. Opole: Wydawnictwo Uniwersytetu Opolskiego, 49-55.

Lipczuk, Ryszard / Misiek, Dorota / Schiewe, Jürgen / Westphal, Werner (Hg.) (2010): Diskurslinguistik - Systemlinguistik. Theorien-Texte-Fallstudien. Hamburg: Dr. Kovač.

Miller, Dorota (2014): Emotionalität und Wertung im Diskurs. Eine kontrastive Analyse deutscher und polnischer Pressetexte zum EU-Beitritt Polens. Studien zur Text-und Diskursforschung. Frankfurt/M.: Lang.

Opiłowski, Roman (2018): Wissenskonstituierung im politischen Online-Diskurs. Deutsche und polnische Tweets zum Präsidenten Donald Trump. In: tekst i dyskurs - text und diskurs 11. Warszawa: Wydawnictwo Uniwersytetu Warszawskiego, 279-294.

Pędzisz, Joanna (2017): Profil des Online-Diskurses in Blog-Interaktionen an der Schnittstelle zwischen theoretischem Konzept und empirischem Modell. Frankfurt/M.: Lang.

Plumpe, Gerhard (1988): Kunst und juristischer Diskurs. Mit einer Vorbemerkung zum Diskursbegriff. In: Fohrmann, Jürgen / Müller, Harro (Hg.): Diskurstheorien und Literaturwissenschaft. Frankfurt/M.: Lang, 330-345. 
Spitzmüller, Jürgen (2005): Metasprachdiskurse. Einstellungen zu Anglizismen und ibre wissenschaftliche Rezeption. Berlin / New York: De Gruyter.

Spitzmüller, Jürgen / Warnke, Ingo (2011): Diskurslinguistik. Eine Einführung in Theorien und Methoden der transtextuellen Sprachanalyse. Berlin / Boston: De Gruyter.

Warnke, Ingo / Spitzmüller, Jürgen (2008): Methoden und Methodologie der Diskurslinguistik Grundlagen und Verfahren einer Sprachwissenschaft jenseits textueller Grenzen. In: Warnke, Ingo / Spitzmüller, Jürgen (Hg.): Methoden der Diskurslinguistik. Sprachwissenschaftliche Zugänge zur transtextuellen Ebene. Berlin / New York: De Gruyter, 3-54.

Wengeler, Martin (2003): Topos und Diskurs. Begründung einer argumentationsanalytischen Methode und ihre Anwendung auf den Migrationsdiskurs (1960-1985). Tübingen: De Gruyter.

Witosz, Bożena (2009): Tekst a/i dyskurs w perspektywie polskiej tradycji badań nad tekstem [Text und Diskurs in der Perspektive der polnischen Tradition der Texterforschung]. In: Bilut-Homplewicz, Zofia / Czachur, Waldemar / Smykała, Marta (Hg.): Lingwistyka tekstu w Polsce i w Niemczech. Pojeccia, problemy, perspektywy [Textlinguistik in Polen und in Deutschland. Begriffe, Probleme, Perspektiven]. Wrocław: ATUT, 19-33.

Witosz, Bożena (2010): Text und/oder Diskurs in der Perspektive der polnischen Tradition der Texterforschung. In: tekst i dyskurs - text und dyskurs 3. Warszawa: Wydawnictwo Uniwersytetu Warszawskiego, 167-179.

Witosz, Bożena (2012): Badania nad dyskursem we wspótczesnym językoznawstwie polonistycznym [Diskursforschung in der gegenwärtigen polonistischen Sprachwissenschaft]. Warszawa: Wydawnictwo Uniwersytetu Warszawskiego.

Witosz, Bożena (2016): Kategoria dyskursu w polonistycznej edukacji akademickiej [Zur Diskurskategorie in der polonistischen akademischen Bildung]. In: Czachur, Waldemar / Kulczyńska, Agnieszka / Kumięga, Łukasz (Hg.): Jak analizować dyskurs? Perspektywy dydaktyczne [Wie soll man den Diskurs analysieren? Didaktische Perspektiven]. Kraków: Universitas, 19-39.

Wodak, Ruth (2005): Sprache und Politik - Sprache in der Politik - Sprache/Sprechen über (Sprache in/und) Politik: Möglichkeiten und Grenzen diskursanalytischer Vorgangsweisen. In: Aptum. Zeitschrift für Sprachkritik und Sprachkultur 2, 135-153.

Wojtak, Maria (2011): O relacjach dyskursu, stylu, gatunku i tekstu [Zu den Relationen zwischen Diskurs, Stil, Gattung und Text]. In: Czachur, Waldemar / Bilut-Homplewicz, Zofia (Hg.): tekst i dyskurs - text und diskurs 3. Warszawa: Wydawnictwo Uniwersytetu Warszawskiego, 69-78.

Żydek-Bednarczuk, Urszula (2005): Wprowadzenie do lingwistycznej analizy tekstu [Einführung in die linguistische Textanalyse]. Kraków: Universitas. 